\title{
EL ENFOQUE REFLEXIVO EN LA FORMACIÓN DOCENTE
}

\section{Tania Tagle ${ }^{1}$}

\author{
RESUMEN
}

El proceso de diseñar un programa focalizado en el desarrollo de la competencia profesional de los docentes es una tarea desafiante. En este marco, el enfoque reflexivo considera algunos elementos que son importantes de tener en cuenta cuando se diseña un programa de estas características, entre los cuales cabe destacar: las creencias de los sujetos en formación, la articulación entre teoría y práctica, así como el propio componente reflexivo.

Palabras clave: formación docente, enfoque reflexivo

\section{THE REFLEXIVE APPROACH TO THE FORMING OF TEACHERS}

\begin{abstract}
The process of designing a Programme focused on developing teachers' professional competence is a challenging task. Within this framework, the reflective approach includes some elements that are important to take into account in this designing process. In relation to this, the reflective approach takes into consideration the following elements: trainees' beliefs, articulation between theory and practice, and reflection.
\end{abstract}

Keywords: teacher training, reflective approach

1 Profesora. Facultad de Educación. Universidad Católica de Temuco. Temuco, Chile. Contacto: ttagle@uct.cl 


\section{EL ENFOQUE REFLEXIVO EN LA FORMACIÓN DOCENTE}

\section{Introducción}

Para responder a los nuevos escenarios en que se mueve la educación es necesario promover el acceso a una formación renovada y de calidad, que les permita a los ciudadanos desarrollar competencias que mejoren sus capacidades productivas para competir en mejores condiciones. En este marco, la calidad de los programas, así como las posibilidades reales que ofrecen a los estudiantes cuando ingresan al mundo laboral, han cobrado alta relevancia demandando, por parte de las instituciones formadoras, cambios de sus modelos educativos.

En el caso de la formación de profesores, el esfuerzo se ha centrado en la búsqueda de propuestas formativas que respondan a los nuevos hallazgos acerca del desarrollo de la competencia profesional y los elementos que la componen. Respecto a esto, las últimas investigaciones en educación superior han puesto de manifiesto la necesidad de desarrollar en los estudiantes posibilidades de aprendizaje y formación tendientes a estimular el pensamiento reflexivo y la reconstrucción del conocimiento.

\section{Modelos de formación docente}

El ejercicio de la profesión docente, en cuanto a su proceso de preparación, ha ido experimentando modificaciones a través del tiempo. Por ejemplo, Martinet, Raymond y Gauthier (2004) identifican cuatro formas básicas asociadas al ejercicio docente: el 'maestro improvisado', 'el maestro artesano', 'el maestro científico' y el 'maestro profesional'. Con respecto al primer tipo de maestro, los autores mencionados establecen que antes del siglo XVII, en Europa, cuando aún en el contexto escolar la educación no había sido formalizada y se dirigía a una audiencia limitada, se pensaba que el conocimiento del contenido disciplinar aseguraba el conocimiento 
de la enseñanza de este; por ejemplo, quien supiese escribir podría enseñar a hacerlo.

Luego, a partir del siglo XVIII comienzan a emerger los primeros indicios de capacitación de los docentes. Así es como, en el proceso de capacitación, se valoraban las 'recetas' del oficio de aquellos reconocidos como los mejores maestros. Según indican Martinet, Raymond y Gauthier (2004, p. 20) "estos trucos y recetas están ordenados en una visión del mundo edificada sobre un control total de los alumnos con objeto de hacerlos seres educados, instruidos y cristianos". Se identifican algunos aportes a la formación de docentes en el sentido que se toma conciencia que contar con el conocimiento disciplinario no asegura el saber enseñarlo y que, entonces, es necesario dominar otros saberes para poder realizar esto, reconociendo que ellos se aprenden. En ese periodo, el aprendizaje se realizaba a través del adiestramiento por parte de un maestro avezado surgiendo así la pedagogía tradicional centrada en este maestro quien ejercía el control absoluto sobre el aprendiz y sobre los contenidos a transmitir.

Continuando con los planteamientos de los especialistas mencionados anteriormente, a fines del siglo XIX y, principalmente, a inicios del siglo XX, se hace extensiva la crítica a la pedagogía tradicional y emerge la 'pedagogía nueva' que da importancia a la ciencia y sugiere una propuesta pedagógica centrada en el niño. En este contexto, surgen con mucho auge los planteamientos de la sicología, emergiendo la idea de hacer de la pedagogía una ciencia y del pedagogo un científico. La pedagogía (como disciplina) se dividió en diferentes escuelas de pensamiento que derivaron a distintos modelos que, de acuerdo a los autores, se pueden clasificar en dos grandes categorías: 'enfoques experimentales' y 'enfoques experienciales'. La primera categoría se asociaría con el positivismo relacionado directamente con el conductismo, y la segunda correspondería a la sicología humanista y sus ramificaciones. En ambos enfoques subyace la idea de que se aprende a enseñar aplicando lo aprendido a la práctica.

Finalmente, la visión de maestro como profesional concibe que este debe acercarse a los requisitos del contexto real de la práctica y 
por lo tanto enfatiza la idea de que el docente aprende en la acción, particularmente a través del análisis de la misma. En este marco, surge el enfoque reflexivo que está basado esencialmente en las ideas de Schön (1983) sobre cómo se desarrolla el expertizaje profesional. Las experiencias prácticas de los estudiantes juegan un rol importante en el proceso de aprender a enseñar, ya que son vistas como la principal fuente del conocimiento profesional (Ur, 2006).

\subsection{El enfoque reflexivo}

Este enfoque incluye algunos elementos que jugarían un rol preponderante en el proceso de desarrollo de la competencia profesional. Estos estarían estrechamente relacionados, y son: los 'esquemas conceptuales existentes' de los estudiantes, el 'conocimiento recibido', el 'conocimiento experiencial', la 'práctica' y la 'reflexión' (Wallace, 2002).

De acuerdo a este modelo, la competencia profesional se desarrolla reflexionando sobre el 'conocimiento recibido' (conocimiento sobre hechos, teorías, etc., que estaría asociado al estudio de una profesión determinada) y el 'conocimiento experiencial' (conocimiento derivado de la experiencia de enseñanza) a la luz de la práctica (Wallace, 2002). A continuación, se presentan cada uno de los componentes incluidos en el modelo reflexivo.

\subsubsection{Creencias}

De acuerdo a Wallace (2002), cuando los alumnos se enrolan en un programa de formación docente ya poseen un conjunto bien establecido de ideas o representaciones sobre la educación. Estas jugarían un rol clave en la forma en la cual los estudiantes interpretan el proceso de enseñanza-aprendizaje y los diferentes elementos que están involucrados en él.

Parece no haber consenso con respecto a qué se entiende por 'creencia', pero sí sobre la relevancia de su estudio. Según Prieto (2007, p. 35) "el debate sostenido sobre el significado más preciso para conceptualizar el fenómeno de las creencias goza de gran importancia en el ámbito educativo, dado que las creencias de los profesores influyen en 
sus percepciones y en sus juicios que, a su vez, determinan su conducta en el aula; de ahí que resulte imprescindible conocer la estructura del sistema de creencias de los profesionales de la educación para mejorar tanto la calidad de su formación como sus prácticas de enseñanza".

Al respecto, las creencias pueden ser entendidas como estructuras cognitivas que representan el sentimiento de certeza que las personas poseen respecto al significado de algo; como tal, se transforman en un filtro de la información que el individuo recoge del entorno, influyendo en el análisis, clasificación e interpretación que de dicha información se realiza (Hamilton, 2005). En su carácter de estructuras cognitivas, las creencias llegan a transformarse en sistemas de explicaciones relativamente coherentes entre sí, que en la interacción con la realidad se constituyen en representaciones o modelos que le permiten al individuo que las posee comprender, explicar y, finalmente, predecir los eventos a su alrededor.

Pajares (1992) afirma que las creencias son 'instrumentales' al seleccionar los instrumentos cognitivos para la interpretación y para el proceso de toma de decisiones, por lo que jugarían un rol importante para definir el comportamiento. Considera que las creencias se muestran inicialmente fragmentadas e inconexas, profundamente arraigadas en la experiencia individual y cotidiana, y que a medida que transcurre el tiempo se transforman, organizándose en estructuras cada vez más coherentes que les permiten a las personas una explicación más acabada de su entorno.

La estructuración y coherencia que alcanzan las creencias permite considerarlas como sistemas que las conectan y relacionan entre sí. La centralidad de una creencia se relaciona directamente con su origen en el tiempo, siendo las más antiguas las que ocupan un lugar más central al interior del sistema y al mismo tiempo se muestran como aquéllas más difíciles de cambiar (Farrell, 2009). La práctica exitosa se constituye en el medio, por excelencia, para su modificación.

En función de las fuentes que influyen en el conocimiento y en las creencias de los docentes, Marcelo (2002, p.27) discute tres categorías de experiencias que influirían en esta construcción: 
- Experiencias personales: incluyen aspectos de la vida que determinan una visión del mundo, creencias hacia uno mismo y en relación con los demás, ideas acerca de las relaciones entre la escuela y la sociedad, así como sobre la familia y la cultura.

- Experiencia con el conocimiento formal: entendido como aquello sobre lo que debe trabajarse en la escuela. Las creencias acerca de la materia que se enseña así como la forma de enseñarla.

- Experiencia escolar y de aula: incluye todas aquellas experiencias como estudiante que contribuyen a formar una idea acerca de qué es enseñar y cuál es el trabajo del profesor.

La idea de que las creencias de los estudiantes sobre la enseñanza y el aprendizaje se desarrollan observando a sus propios profesores desempeñando su rol profesional ha sido discutida por diferentes investigadores (ver, por ejemplo, Blázquez y Tagle, 2010). Se ha argumentado que estas creencias son extremadamente difíciles de cambiar y jugarían un rol preponderante en cómo los estudiantes interpretan nuevas ideas sobre el aprendizaje y la enseñanza, y en cómo esas ideas son trasladadas a la práctica.

Si se considera que las creencias actúan como filtros de la nueva información podría, a su vez, considerarse que su modificación sería un propósito que no se puede alcanzar, pues la persona continuamente dejaría de lado aquella información opuesta a sus creencias. Pero esto no opera de forma radical ya que se ha demostrado que, en el tiempo, las creencias pueden cambiarse a través de la experiencia, involucrando nuevas prácticas pedagógicas. Asociado a este punto, Brown (2004) indica que las creencias de los estudiantes de pedagogía que han sido construidas a partir de experiencias previas a la formación profesional se pueden modificar a través de un proceso de reflexión de las mismas y de experiencias que emergen en su desarrollo como profesionales de la educación.

Un factor que parece afectar el desarrollo o cambio de las creencias previas de los estudiantes durante el proceso de formación profesional dice relación con el carácter implícito de éstas. Se ha planteado que las creencias usualmente permanecen a un nivel tácito, y por lo tanto son difíciles de articular y explorar, y este hecho, 
a su vez, explicaría la perpetuación de las prácticas pedagógicas tradicionales (Pajares, 1992; Vacilotto y Cummins, 2007).

Diferentes investigadores que han llevado a cabo estudios focalizados en investigar las creencias de los estudiantes de pedagogía (ver, por ejemplo, Thomas y Pedersen, 2003; Davis, 2003) han sugerido que sus hallazgos pueden tener algunas implicancias para el proceso de preparación de profesores. Entre estas se pueden identificar las siguientes: Primera, se ha sugerido que los formadores de educadores necesitan comprender la naturaleza de las creencias de los estudiantes para poder diseñar estrategias de formación adecuadas que les ayuden a desarrollar sus creencias más efectivamente. Segunda, se ha señalado que si los formadores de educadores son conscientes de los tipos de creencias que los estudiantes han construido cuando ingresan a su proceso de formación profesional, pueden seleccionar con mayor facilidad el contenido de un curso de formación de docentes e identificar algunas actividades que les ayuden a desarrollar sus creencias previas. Tercera, se ha planteado que los formadores de educadores necesitan considerar que el proceso de aprender será más significativo si se construye a partir de las creencias de los estudiantes. Al respecto, Malderez (2002) indica que cuando se les ha solicitado a los estudiantes identificar las características de una 'buena enseñanza' que promueva el aprendizaje, ellos invariablemente han mencionado a profesores que, independientemente de los métodos utilizados, han considerado como punto de partida sus conocimientos previos, experiencias y creencias, y los han ayudado a articular los mismos con la nueva información. Hart (2002) señala que es imperativo que los programas de formación de docentes evalúen su efectividad, al menos en parte, considerando cuán bien ellos nutren a los estudiantes con creencias que sean consistentes con la filosofía de aprendizaje y de enseñanza del programa. Finalmente, Hayes (2004) indica que las creencias de los alumnos requieren un nivel de reflexión para contribuir a la construcción teórica del proceso de enseñanza y aprendizaje en la formación profesional.

\subsubsection{Integración de la teoría y la práctica}

La relación que se establece entre la teoría y la práctica en el proceso de formación del profesorado ha sido un tema discutido por diferentes 
autores (ver, por ejemplo, Blázquez, 2000). Al respecto, se ha señalado que el principal problema es que la teoría y la práctica son vistas como componentes separados en el proceso de preparación de profesionales; esto es, la teoría es vista como una generalización abstracta que no tiene conexión obvia con la realidad de la enseñanza, y la práctica es percibida como acciones implementadas en la sala de clases (Ur, 2006). Esta visión particular sobre la teoría y la práctica estaría basada en un enfoque específico sobre el proceso de educación profesional llamado 'racionalidad técnica' (Schön, 1983).

De acuerdo con Schön (1983), el modelo de racionalidad técnica ha ejercido una poderosa influencia en la forma de entender el desarrollo del conocimiento profesional, y por lo tanto, en la manera en la que diferentes profesionales, entre ellos los profesores, han sido formados en las instituciones educacionales. Este modelo asume que el principal objetivo de cualquier actividad profesional es instrumental, es decir, la preocupación de cualquier acción profesional es resolver problemas prácticos o lograr algunos objetivos aplicando la teoría derivada de la investigación científica. Este modelo sugiere una separación entre teoría y práctica en el proceso de preparación profesional y hace una distinción entre dos tipos de conocimiento: uno derivado del estudio científico y el otro derivado de la experiencia práctica. Respecto a este punto, Schön plantea que en este modelo la investigación se presenta institucionalmente separada de la práctica y se conecta con esta por relaciones de intercambio cuidadosamente definidas.

Estrechamente asociado a lo discutido con antelación, Korthagen (2001) indica que los programas de formación de profesores 'tradicionales' están basados generalmente en tres presupuestos que generarían una brecha entre la teoría y la práctica: a) las teorías ayudan a los profesores a desempeñarse mejor en su profesión; b) las teorías deben estar basadas en investigación científica, y c) los formadores de profesores deberían hacer una elección respecto a las teorías que deberían ser incluidas en los programas de formación.

Con relación a la información presentada en los párrafos previos, se ha argumentado que el principal problema derivado del modelo 
de 'racionalidad técnica' no está asociado con la importancia dada al componente teórico del proceso de preparación profesional, sino con la relación entre teoría y práctica que es, implícita o explícitamente, expresada en este modelo. Al respecto, Blázquez (2000) indica que la formación profesional de un docente se fundamenta en una estrecha relación entre los componentes teórico y práctico, donde sin este último la teoría es inútil, y viceversa. El autor antes mencionado alude a una visión dialéctica para explicar la relación entre teoría y práctica, indicando que existe indisociabilidad entre ambos componentes ya que la práctica es teoría en acción.

El modelo reflexivo reconoce esta interacción entre teoría y práctica y estima que el 'conocimiento recibido' debería informar al 'conocimiento experiencial' y, a su vez, debería ser directamente informado por éste (Wallace, 2002). Es decir, la teoría informa la práctica y el proceso de reflexión sobre la práctica modifica la teoría (teoría y práctica siempre presentarían una estrecha relación en el proceso de desarrollo profesional).

\subsubsection{Reflexión}

Parece haber acuerdo sobre el importante rol que juega la reflexión en el proceso de formación profesional (ver; por ejemplo, Malderez y Wedell, 2007). Sin embargo, algunos autores señalan que el significado del término 'reflexión' y las estrategias para promover su desarrollo son temas complicados (por ejemplo, Kumpulainen, Toom y Saalasti, 2009). Si el lector tiene la posibilidad de revisar algunos estudios focalizados en el proceso de reflexión y en las estrategias para favorecer su desarrollo, él o ella encontrará que el término 'reflexión' es definido de formas distintas por diferentes especialistas. La mayoría de las definiciones están basadas en las ideas de Dewey (1933) y/o Schön $(1983,1987)$, quienes han ejercido una influencia importante en las formas en las que el proceso de reflexión es actualmente entendido.

Dewey, en su libro How We Think (1933), define la reflexión como una cuidadosa, persistente y activa consideración de cualquier creencia o forma de conocimiento a la luz de los cimientos que la sustentan. De acuerdo con Dewey (1933), hay dos elementos involucrados en la reflexión: a) un estado de incertidumbre, y b) un proceso de 
investigación que se centra en desvelar algunos factores que pueden validar o no una forma particular de conocimiento o creencia.

Por su parte Donald Schön, en sus libros: The Reflective Practitioner (1983) y Educating the Reflective Practitioner (1987), presenta su visión particular sobre el rol que la reflexión jugaría en el proceso de desarrollo profesional. Sugiere que cuando cualquier profesional enfrenta una situación inesperada en su actuar, tiende a reflexionar sobre los fundamentos que subyacen a esa situación, y al hacer esto, llegaría a nuevas comprensiones de sus acciones y las modificaría.

En este punto, es importante indicar que Dewey y Schön parecen coincidir en que la reflexión es una forma de pensamiento deliberado y consciente la cual involucra un cuidadoso análisis de presupuestos, ideas y conocimientos. De acuerdo a estos autores, la reflexión y la acción estarían estrechamente relacionadas, esto es, la reflexión estaría cimentada en la acción o experiencia y, al mismo tiempo, la modificaría.

Con respecto a lo anterior, es relevante mencionar que algunos especialistas plantean que a pesar de la importancia dada a la reflexión en el desarrollo profesional de los docentes, no todos ellos la practican, algunos ni siquiera tienen claridad del beneficio de este proceso (ver por ejemplo, Prieto, 2007). Entre los factores que se asociarían a la incapacidad de reflexionar están: la falta de motivación profesional, el probable desconocimiento del proceso de reflexión en el mejoramiento de la enseñanza y/o el temor que puede existir para afrontar riesgos o ciertos elementos del contexto de desempeño (Prieto, 2007).

\subsubsection{Competencia profesional}

La competencia profesional de los docentes está estrechamente relacionada con el proceso de toma de decisiones apropiadas en el ámbito pedagógico. Respecto a esta, Ávalos (2004) indica que el concepto competencia se refiere concretamente al desempeño y específicamente para los docentes estaríamos aludiendo a una actividad dirigida a estimular cambios en los otros. No se habla de una conducta específica sino de una cualidad del trabajo docente que se tiene en menor o mayor grado. 
Martinet, Raymond y Gauthier (2004) indican que la literatura plantea un número de características asociadas al concepto competencia. Algunas de éstas son:

- La competencia se ejercita en un contexto real. Se desarrolla en un contexto determinado con todas las variables que éste involucra.

- La competencia se ubica en un continuo que va de lo sencillo a lo complejo. Es aconsejable realizar una progresión en cuanto al nivel de logro de las competencias que se espera que los estudiantes obtengan en diferentes etapas del proceso de formación profesional.

- La competencia pertenece al orden del 'saber movilizar' en el quehacer profesional. Si bien es cierto se requiere disponer de los distintos tipos de saberes como recursos para fundamentar la competencia, se precisa de un contexto real para identificar la realización de la misma. Es así como se exige que el individuo competente sepa interpretar y analizar la situación, detectar los recursos disponibles y ejecutar una acción, integrando efectivamente y en forma pertinente todos los elementos.

- La competencia es un saber-actuar logrado, eficaz, ágil e inmediato que se manifiesta en forma recurrente. La competencia se expresa por una ejecución expedita, realizándose de manera recurrente en diversas situaciones, indicando que el saber-actuar está estabilizado

\section{Comentarios finales}

En síntesis, el proceso de diseño de un programa destinado a la formación de docentes es una tarea, sin duda, compleja. Al respecto, se sugiere tener en consideración los principios asociados al enfoque reflexivo (la explicitación y cuestionamiento de las 'creencias' de los estudiantes; la articulación entre la teoría y la práctica; y la generación de espacios para la reflexión sobre y en la acción) cuando se diseña un programa focalizado en el desarrollo de la competencia profesional de los profesores.

Al considerarse los elementos anteriormente mencionados, y si se reconoce que las creencias de los estudiantes funcionan como filtros para procesar la nueva información, los programas de formación de profesores necesitan proveer a los estudiantes de oportunidades para hacer explícitas sus creencias personales y examinarlas. Asimismo, las 
creencias pueden modificarse si son cuestionadas a través del proceso de formación profesional, reconociendo que éstas no son útiles para enfrentar las demandas del contexto actual.

La práctica progresiva como actividad curricular incluida en el proceso formativo de los estudiantes de pedagogía juega un rol importante en el proceso de modificación de las creencias de los mismos en la medida que genera instancias para la acción profesional y para la reflexión de esa acción, pero el análisis reflexivo es una tarea desafiante que no se desarrolla naturalmente. Este análisis tiene que estar basado en las experiencias prácticas de los estudiantes en formación.

\section{Referencias bibliográficas}

Ávalos, B. (2004). Las instituciones formadoras de docentes y las claves para formar buenos docentes. Santiago: Ministerio de Educación de Chile.

Blázquez, F. (2000). Las funciones del tutor en el centro educativo. Campo Abierto 18, 69-86.

Blázquez, F. y Tagle, T. (2010). Formación docente: Un estudio de las creencias de alumnos y profesores sobre el proceso de enseñanza y aprendizaje del inglés. Revista Iberoamericana de Educación n. ${ }^{\circ}$ 54/4, pp. 1-12.

Brown, R. (2004). Beliefs, concepts and the training of trainers. En D. Hayes, (Ed.) Trainer development: Principles and practice from language teacher training. Melbourne: Language Australia.

Davis, A. (2003). Teachers' and students' beliefs regarding aspects of language learning. Evaluation and Research in Education. Vol. 17, n. ${ }^{\circ} 4$.

Dewey, J. (1933). How we think. London: D.C. Health Co.

Farrell, T. (2009). Critical reflection in a TESL course: Mapping conceptual change. ELT Journal 63, pp. 221-229.

Hamilton, D. (2005). Social cognition. New York: Psychology Press.

Hart, L. (2002). Preservice teachers' beliefs and practice after participating in an integrated content/ methods course. School Science and Mathematics Vol. 102-1.

Hayes, D. (2004). Trainer development: Principles and practice for language teacher training. Melbourne: Language Australia.

Korthagen, F. (2001). Teacher education: A problematic enterprise. En F. A. J Korthagen, J. Kessels, B. Koster, B. Langerwerf, y T. Wubbels (Eds.), 
Linking practice and theory: the pedagogy of realistic teacher education. New Jersey: Lawrence Erlbaum Associates, Publishers.

Kumpulainen, K., Toom, A. y Saalasti, M. (2009). Video as cultural landscape for reflection and identity work in teacher education. En M. César y K. Kumpulainen (Eds.) Social interactions in multicultural settings. Rotterdam: Sense Publishers.

Malderez, A. (2002). Mentor courses: A resource book for trainer-trainers. Cambridge: Cambridge University Press.

Malderez, A. y Wedell, M. (2007). Teaching teachers: Processes and practices. London: Continuum.

Marcelo, C. M. (2002). Aprender a enseñar para la sociedad del conocimiento. EPPA Vol 10.

Martinet, M.; Raymond, D. y Gauthier C. (2004). Formación de docentes: Orientaciones, competencias profesionales. Québec: Ministerio de Educación de Québec.

Pajares, M. F. (1992). Teachers' beliefs and educational research: Cleaning up a messy construct. Review of Educational Research 62/3, 307-332.

Prieto, L. (2007). Autoeficacia del profesor universitario: eficacia percibida y práctica docente. Madrid: Narcea Ediciones.

Schön, D. (1983). The reflective practitioner: how professionals think in action. London: Temple Smith.

Schön, D. (1987). Educating the reflective practitioner: toward a new design for teaching and learning in the professions. San Francisco: Jossey-Bass Publishers.

Thomas, J. y Pedersen, J. (2003). Reforming elementary science teacher preparation: what about extant teaching beliefs. School Science $\&$ Mathematics. Vol. 103, 7.

Ur, P. (2006). A course in language teaching: Practice and theory. Cambridge: Cambridge University Press.

Vacilotto, S. y Cummings, R. (2007). Peer coaching in TEFL/TESL programmes. ELT Journal 61/2, pp. 153-160.

Wallace, M. (2002). Training foreign language teachers: A reflective approach. Cambridge: Cambridge University Press. 